\title{
Finite-Window RLS Algorithms
}

This paper was downloaded from TechRxiv (https://www.techrxiv.org).

\section{LICENSE}

CC BY 4.0

SUBMISSION DATE / POSTED DATE

$15-02-2022$ / 22-02-2022

CITATION

Shen, Lu; Zakharov, Yuriy; Niedźwiecki, Maciej; Gańcza, Artur (2022): Finite-Window RLS Algorithms. TechRxiv. Preprint. https://doi.org/10.36227/techrxiv.19175525.v1

$\mathrm{DOI}$

10.36227/techrxiv.19175525.v1 


\title{
Finite-Window RLS Algorithms
}

\author{
Lu Shen, Member, IEEE, Yuriy Zakharov, Senior Member, IEEE \\ Maciej Niedźwiecki, Senior Member, IEEE and Artur Gańcza, Student Member, IEEE
}

\begin{abstract}
Two recursive least-squares (RLS) adaptive filtering algorithms are most often used in practice, the exponential and sliding (rectangular) window RLS algorithms. This popularity is mainly due to existence of low-complexity versions of these algorithms. However, these two windows are not always the best choice for identification of fast time-varying systems, when the identification performance is most important. In this paper, we show how RLS algorithms with arbitrary finite length windows can be implemented at a complexity comparable to that of exponential and sliding window RLS algorithms. Then, as an example, we show an improvement in the performance when using the proposed finite-window RLS algorithm with the Hanning window for identification of fast time-varying systems.
\end{abstract}

Index Terms-Adaptive filter, finite-window, RLS, time-varying systems

\section{INTRODUCTION}

In the adaptive filtering, recursive least-squares (RLS) algorithms are very popular. They possess fast convergence, while the complexity and numerical stability of their implementation can be comparable to that of slower convergent algorithms, such as the least-mean squares algorithms. An RLS algorithm, at every time instant $n$ minimises the cost function

$$
J_{w}(\mathbf{h})=\sum_{i=-\infty}^{\infty} w(i-n)|e(i)|^{2} \rightarrow \min _{\mathbf{h}}
$$

where $e(i)=z(i)-\mathbf{h}^{H} \mathbf{x}(i)$ is the error signal, $z(i)$ is the desired signal, $\mathbf{x}(i)=[x(i), x(i-1), \ldots, x(i-L+1)]^{T}$ is the $L \times 1$ regressor vector, and $x(i)$ is the adaptive filter input.

Two RLS algorithms are most often used in practice, the exponential window (ERLS) and sliding window (SRLS) algorithms. The ERLS exploits the (infinite in length) window $w(i): w_{\text {ERLS }}(i)=\lambda^{-i}$ for $i \in(-\infty, 0]$ and $w_{\text {ERLS }}(i)=0$ otherwise, where $0<\lambda<1$ is the forgetting factor, a parameter defining the efficient length of the window. The SRLS uses the finite window $w(i): w_{\text {SRLS }}(i)=1$ for $i \in[-M+1,0]$ and $w_{\text {SRLS }}(i)=0$ otherwise, where $M$ is the length of the (rectangular) sliding window. Using the matrix inversion formula for a low-rank matrix update, these two windows allow the RLS algorithm implementation with a complexity of $\mathcal{O}\left(L^{2}\right)$ arithmetic operations per time instant [1], [2]. The complexity can be further reduced to $\mathcal{O}(L)$ arithmetic operations [2]-[4]. However, as indicated in [7], identification of time-varying systems can benefit from using symmetric bell-shaped windows, like the Hamming, Hanning, Parzen, Bartlett, and other windows [5], [6]. The main problem with these windows is the high complexity,

The work of L. Shen and Y. Zakharov was supported in part by the U.K. EPSRC through Grants EP/P017975/1 and EP/R003297/1. The work of M. Niedźwiecki and A. Gańcza was partially supported by the National Science Center under the agreement UMO-2018/29/B/ST7/00325. which in general is $\mathcal{O}\left(L^{3}\right)$ or higher. In this letter, we show that RLS algorithms with an arbitrary finite window $w(i)$ of length $M$ can be implemented with a complexity dominated by the term $\mathcal{O}\left((M+L) \log _{2}(M+L)\right)$. This is achieved by using the fast Fourier transform (FFT) and dichotomous coordinate descent (DCD) iterations [4], [8], [9]. We then demonstrate that other windows, as an example - the Hanning window, can provide a significant improvement in performance when identifying time-varying systems, compared to the exponential and rectangular windows.

When identifying time-varying systems, the excellent performance is achieved by adaptive filters based on the local basis function (LBF) principle [10]. However, such filters are too complicated for practice, with a complexity of at least $\mathcal{O}\left(P^{3} L^{3}\right)$, where $P$ is the number of basis functions. A similar performance with much lower complexity can be achieved by fast LBF (fLBF) adaptive filters exploiting two stages, preestimation and post-filtering [11]. The pre-estimation stage provides maximum bandwidth parameter estimates which are almost unbiased but with a large variability. Therefore, denoising is carried out at the post-filtering stage [11]. However, there is still some bias introduced in the pre-estimation stage which limits the overall performance of fLBF algorithms when identifying fast time-varying systems. Meanwhile, the dominant complexity component is brought in by ERLS adaptive filtering. In this letter, we show that the finite-window RLS (FRLS) adaptive filter as the pre-estimator can significantly improve the performance of fLBF algorithms.

This letter is organized as follows. In Section II, the FRLS algorithm is derived. Section III introduces the fLBF algorithm. The identification performance of the FRLS algorithm is demonstrated by numerical simulation in Section IV. The letter is concluded in Section V.

Notations: In this letter, we use capital and small bold fonts for matrices and vectors, e.g. $\mathbf{R}$ and $\mathbf{h}$, respectively. We denote the complex conjugate as $(\cdot)^{*}$, transpose of $\mathbf{h}$ as $\mathbf{h}^{T}$, and the Hermitian transpose of $\mathbf{h}$ as $\mathbf{h}^{H}$. The $p$ th column of matrix $\mathbf{R}$ is denoted as $\mathbf{R}^{(p)}$. The norm of a vector is denoted as $\|\cdot\|$, the element-wise product of two vectors $\mathbf{u}$ and $\mathbf{v}$ is denoted as $\mathbf{u} \odot \mathbf{v}$, and the Kronecker product of two vectors $\mathbf{u}$ and $\mathbf{v}$ is denoted as $\mathbf{u} \otimes \mathbf{v}$.

\section{FRLS ADAPTIVE ALGORITHM}

The minimization of the cost function in (1), for a window $w(i)$ defined on a finite support $\Gamma$, results in the solution

$$
\hat{\mathbf{h}}(i)=\mathbf{R}^{-1}(i) \boldsymbol{\beta}(i),
$$

where

$$
\mathbf{R}(i)=\sum_{k \in \Gamma} w(k) \mathbf{x}(i+k) \mathbf{x}^{H}(i+k)
$$


TABLE I: FRLS algorithm

\begin{tabular}{|c|c|}
\hline Step & Equation \\
\hline & $\begin{array}{l}\text { for } i<0: \mathbf{x}(i)=\mathbf{0}, \hat{\mathbf{h}}(i)=\mathbf{0} \\
\mathbf{R}(i)=\mathbf{0}, \mathbf{z}(i)=\mathbf{0} \\
\text { for } i=0,1, \ldots, N\end{array}$ \\
\hline 1 & $\begin{array}{l}\text { Computing the first column of } \mathbf{R}(i) \\
\mathbf{s}_{w}=\operatorname{FFT}\{\tilde{\tilde{w}}(i)\} \\
\mathbf{s}_{x}=\operatorname{FFT}\left\{\tilde{\mathbf{x}}^{*}(i)\right\} \\
\mathbf{R}^{(1)}(i)=\operatorname{IFFT}\left\{\mathbf{s}_{w} \odot \mathbf{s}_{x}\right\}\end{array}$ \\
\hline 2 & $\begin{array}{l}\text { Computing the filter output } y(i, k) \text { for } k \in \Gamma \text { : } \\
\mathbf{s}_{h}=\operatorname{FFT}\{\mathbf{h}(i-1)\} \\
\mathbf{y}(i)=\operatorname{IFFT}\left\{\mathbf{s}_{x} \odot \mathbf{s}_{h}\right\}\end{array}$ \\
\hline 3 & $\begin{array}{l}\text { Computing the error signal: } \\
\mathbf{e}(i)=\mathbf{z}(i)-\mathbf{y}(i)\end{array}$ \\
\hline 4 & $\begin{array}{l}\text { Computing the residual vector: } \\
\mathbf{e}_{\mathbf{w}}(i)=\mathbf{e}^{*}(i) \odot \mathbf{w} \\
\mathbf{s}_{e}=\operatorname{FFT}\left\{\mathbf{e}_{\mathbf{w}}(i)\right\} \\
\mathbf{r}(i)=\left[\operatorname{IFFT}\left\{\mathbf{s}_{e} \odot \mathbf{s}_{x}\right\}\right]^{*}\end{array}$ \\
\hline 6 & Solve (8) with DCD iterations \\
\hline 7 & $\hat{\mathbf{h}}(i)=\hat{\mathbf{h}}(i-1)+\Delta \hat{\mathbf{h}}(i)$ \\
\hline
\end{tabular}

is the $L \times L$ regression matrix, and

$$
\boldsymbol{\beta}(i)=\sum_{k \in \Gamma} w(k) \mathbf{x}(i+k) z^{*}(i+k)
$$

is the $L \times 1$ cross-correlation vector. The support $\Gamma$ for the ERLS algorithm is $\Gamma=[-\infty, 0]$. The support for the SRLS algorithm is $\Gamma=[-M+1,0]$; for the delayed SRLS (SRLSd) algorithm [12], which is a non-causal version of the SRLS algorithm, it is $\Gamma=\left[-M_{0}, M_{0}\right]$, where $M=2 M_{0}+1$; etc.

Thus, finding the solution in (2), for every time instance $i$, requires computation of elements of the matrix $\mathbf{R}(i)$ and its inversion, which are most complicated steps of the algorithm.

Since $\mathbf{R}(i)$ is a Hermitian matrix, the direct computation in (3) requires about $2 M L^{2}$ real-valued multiply and accumulate (MAC) operations, where it is also taken into account that a complex-valued multiplication requires 4 realvalued multiplications. For the transversal-structured regressor as in (1), the following relationship can be used for updating $\mathbf{R}(i)$ (see proof in [13]):

$$
[\mathbf{R}(i)]_{m+1, n+1}=[\mathbf{R}(i-1)]_{m, n},
$$

where $\mathbf{R}_{m, n}$ denotes an element of the matrix $\mathbf{R}$ at the $m$ th column and $n$th row, and $m, n=1, \ldots, L-1$. Note that only the first column $\mathbf{R}^{(1)}(i)$ of $\mathbf{R}(i)$ needs an update, which with the direct computation requires $4 M L$ operations, this still can be high. However, the complexity of the update can be reduced if using the FFT as follows. The first column of $\mathbf{R}(i)$ is given by

$$
\begin{aligned}
\mathbf{R}^{(1)}(i) & =\sum_{k \in \Gamma} w(k) x(i+k) \mathbf{x}^{*}(i+k) \\
& =\sum_{k \in \Gamma} \tilde{w}(i, k) \mathbf{x}^{*}(i+k),
\end{aligned}
$$

where $\tilde{w}(i, k)=w(k) x(i+k), k \in \Gamma$, are elements of an $M$-length vector $\tilde{\mathbf{w}}(i)$. The $L$-length column $\mathbf{R}^{(1)}(i)$ can be considered as convolution of the $M$-length sequence of elements in $\tilde{\mathbf{w}}(i)$ and the $(M+L)$-length sequence of all elements in the vector $\tilde{\mathbf{x}}^{*}(i)=\left[x^{*}\left(i-M_{0}-L\right), \ldots, x^{*}(i+\right.$ $\left.\left.\left.M_{0}\right)\right)\right]^{T}$. The convolution can be efficiently computed in the frequency domain. Specifically, the sequence $\tilde{w}(k)$ is zeropadded to the length $M+L$, FFT $\mathbf{s}_{w}$ of $\tilde{\mathbf{w}}(i)$ and FFT $\mathbf{s}_{x}$ of $\tilde{\mathbf{x}}^{*}(i)$ of $(M+L)$-length are computed, the element-wise product $\mathbf{s}_{w} \odot \mathbf{s}_{x}$ is computed, and the inverse FFT (IFFT) of $\mathbf{s}_{w} \odot \mathbf{s}_{x}$ is finally computed, its last $L$ elements represent the column $\mathbf{R}^{(1)}(i)$. Therefore, the complexity is reduced from $4 M L$ operations to three FFTs of size $(M+L)$, which is $\mathcal{O}\left((M+L) \log _{2}(M+L)\right)$. Another important property of this approach is that the computation of $\mathbf{R}(i)$ is numerically stable, since no recursion is required.

The rest of the algorithm complexity is dominated by computation in (2). For the numerical stability of this computation, it is preferable to avoid the matrix inversion, which is also a computationally demanding operation. Therefore, instead of the matrix inversion and matrix-vector multiplication in (2), we solve the normal equation

$$
\mathbf{R}(i) \mathbf{h}(i)=\boldsymbol{\beta}(i),
$$

and obtain a (possibly approximate) solution $\hat{\mathbf{h}}(i)$. A direct (precise) solution, e.g., using the Cholesky decomposition, would require $\mathcal{O}\left(L^{3}\right)$ MACs. This can be reduced as follows. Instead of solving the system of equations in (7), we can solve:

$$
\mathbf{R}(i) \Delta \mathbf{h}(i)=\mathbf{r}(i),
$$

where

$$
\mathbf{r}(i)=\boldsymbol{\beta}(i)-\mathbf{R}(i) \hat{\mathbf{h}}(i-1)
$$

is a residual vector for instance $i$ when using the solution $\hat{\mathbf{h}}(i-1)$ obtained at the previous instance $i-1$. The solution to (7) for instance $i$ is then found as

$$
\hat{\mathbf{h}}(i)=\hat{\mathbf{h}}(i-1)+\Delta \hat{\mathbf{h}}(i),
$$

where $\Delta \hat{\mathbf{h}}(i)$ is an approximate solution to (8). The benefit of this approach is that a solution to (8) can be found with a few simple iterations, e.g., such as the DCD iterations [8].

With this approach, the most computationally demanding step in (9) can be simplified if we take into account that, as follows from (3) and (4),

$$
\mathbf{r}(i)=\sum_{k \in \Gamma} w(k) e^{*}(i, k) \mathbf{x}(i+k),
$$

where $e(i, k)=z(i+k)-y(i, k)$ is an error signal, and $y(i, k)=\hat{\mathbf{h}}^{H}(i-1) \mathbf{x}(i+k)$. We denote $\mathbf{y}(i)$ a vector with elements $y(i, k), k \in \Gamma$. These elements represent an $M$-length sequence, which is a convolution of the $L$-length sequence of elements in $\hat{\mathbf{h}}(i-1)$ and the $(M+L)$-length sequence of all elements in $\tilde{\mathbf{x}}(i)$. Since the FFT $\mathbf{s}_{x}$ of $\tilde{\mathbf{x}}^{*}(i)$ is already available, the computation of $\mathbf{y}(i)$ requires one FFT and one IFFT of length $(M+L)$. The error vector $\mathbf{e}(i)$ with elements $e(i, k), k \in \Gamma$, is then given by $\mathbf{e}(i)=\mathbf{z}(i)-\mathbf{y}(i)$, where $\mathbf{z}(i)$ is a vector with elements $z(i+k), k \in \Gamma$. After computing the vector $\mathbf{e}(i)$, the residual vector $\mathbf{r}(i)$ in (11) can be computed similarly to (6), but, with the available FFT $\mathbf{s}_{x}$, using only one FFT and one IFFT.

The system of equations in (8) can be solved using the leading DCD algorithm (see details in [4]). At every iteration, the DCD algorithm updates one element of the vector $\Delta \hat{\mathbf{h}}(i)$ 
corresponding to the element of $\mathbf{r}(i)$ with the maximum magnitude. Four directions of update, $[-1,1,-j, j], j=\sqrt{-1}$, are analysed, and if the cost function can be minimized, the direction providing the minimum is chosen; such an iteration is called successful. If the cost function cannot be reduced after searching over all four directions, the step size is reduced by two. The initial step size $H$ is chosen as a power of two for efficient hardware implementation. The maximum number of times $M_{b}$ the step size can be reduced is equivalent to the number of bits representing the solution vector. The DCD algorithm requires no multiplication or division and its complexity depends on the number of successful DCD iterations $N_{u}$. When the system parameters are time-invariant or slowly time-varying, a small number of updates $\left(N_{u}=1,2\right)$ can be used. For fast time-varying channels, a higher number of updates is required; in our examples below, we set $N_{u}=8$.

The FRLS algorithm is summarized in Table I. Its complexity is $\left(18+4 N_{u}\right) L+16 M+M_{b}$ MACs and 7 FFTs of size $M+L$.

\section{III. fLBF ALGORITHM WITH FRLS PRE-ESTIMATOR}

The fLBF algorithm includes two stages [11]. At the first stage, pre-estimation is carried out to provide unbiased estimates of the system taps.

A popular pre-estimate $\tilde{\mathbf{h}}(i)$ is the result of inverse filtering

$$
\tilde{\mathbf{h}}(i)=\frac{1}{1-\lambda}\left[\hat{\mathbf{h}}_{\mathrm{ERLS}}(i)-\lambda \hat{\mathbf{h}}_{\mathrm{ERLS}}(i-1)\right]
$$

of estimates $\hat{\mathbf{h}}_{\mathrm{ERLS}}(i)$ obtained by the ERLS adaptive filter; the inverse filtering in (12) is applied to reduce the bias of the pre-estimates [11]. At the second stage of the fLBF algorithm, the pre-estimates are post-filtered to reduce the estimation variance, while keeping the bias small:

$$
\begin{aligned}
& \hat{\mathbf{h}}_{\mathrm{fLBF}}(i)=\mathbf{F}(0) \hat{\boldsymbol{\alpha}}_{\mathrm{fLBF}}(i), \\
& \hat{\boldsymbol{\alpha}}_{\mathrm{fLBF}}(i)=\sum_{k=-K_{0}}^{K_{0}} \mathbf{F}^{H}(k) \tilde{\mathbf{h}}(i+k),
\end{aligned}
$$

where $\mathbf{F}(k)=\mathbf{I}_{L} \otimes \mathbf{f}(k), \mathbf{f}(k)=\left[f_{1}(k), \ldots, f_{P}(k)\right]^{T}$ is the vector of orthogonal basis functions $f_{p}(\cdot)$, defined on a time interval $\left[-K_{0}, K_{0}\right]$, and $P$ is the number of basis functions. In this letter, we adopt the complex exponential basis set of the form (see [14]-[16] for a physical justification of such a choice in application to fast-varying communication channels)

$$
\begin{aligned}
& \left\{f_{1}(j), \ldots, f_{P}(j), j \in\left[-K_{0}, K_{0}\right]\right\} \\
& \quad=\left\{\frac{1}{\sqrt{K}} e^{\mathrm{i} j \omega_{1}}, \ldots, \frac{1}{\sqrt{K}} e^{\mathrm{i} j \omega_{P}}, j \in\left[-K_{0}, K_{0}\right]\right\},
\end{aligned}
$$

where $\mathrm{i}=\sqrt{-1}, \omega_{1}=0, P=2 m_{0}+1, K=2 K_{0}+1$, and

$$
\omega_{2 l}=-\frac{2 \pi l}{K}, \quad \omega_{2 l+1}=\frac{2 \pi l}{K}, \quad l=1, \ldots, m_{0} .
$$

The complexity of the fLBF algorithm is dominated by the ERLS adaptive filtering.

The fLBF algorithm shows high identification performance in time-varying scenarios. However, the performance can be further improved with a better pre-estimator. For this purpose,

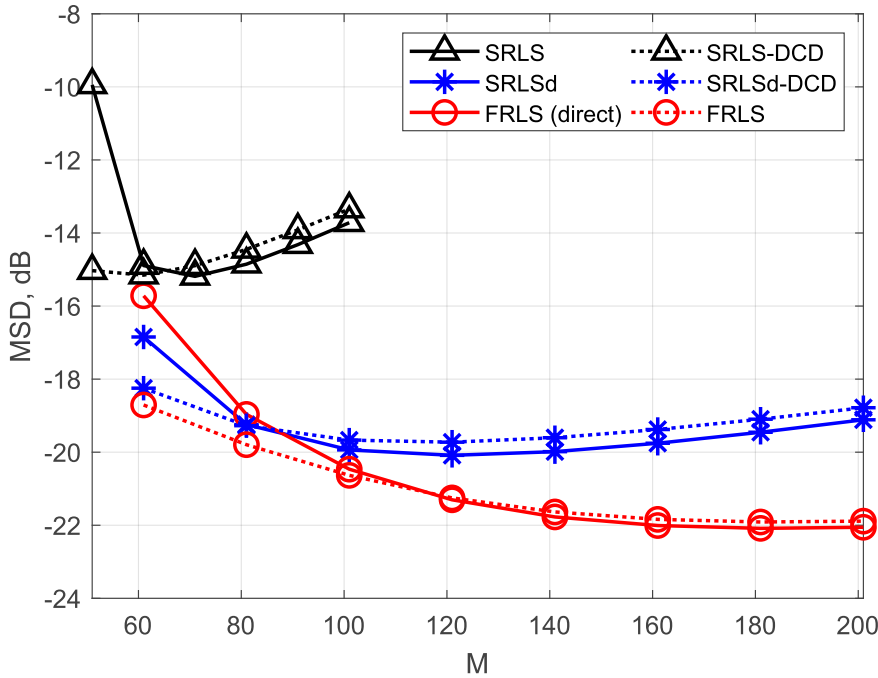

Fig. 1: MSD performance of SRLS and FRLS algorithms when identifying time-varying systems; SNR $=25 \mathrm{~dB}$. Note that FRLS (direct) is the version with the direct solution (2).

we propose to use the FRLS algorithm, so that $\tilde{\mathbf{h}}(i)=$ $\hat{\mathbf{h}}_{\mathrm{FRLS}}(i)$.

\section{Numerical Results}

In this section, we compare the identification performance and complexity of the FRLS algorithm with the Hanning window on the support $\Gamma=\left[-M_{0}, M_{0}\right]$ with that of other algorithms. We consider the following signal model:

$$
z(i)=\mathbf{h}^{H}(i) \mathbf{x}(i)+n(i),
$$

where $\mathbf{h}(i)$ is a time-varying impulse response of an unknown system to be identified, $\mathbf{x}(i)$ is the regressor vector with zero-mean uncorrelated complex-valued Gaussian numbers of unit variance and $n(i)$ is a zero-mean complex-valued white Gaussian noise. The $L=50$ system taps are modelled as independent zero-mean unit-variance random processes with a uniform power spectral density within the frequency interval $\left[-f_{\max }, f_{\max }\right]$. Realizations of the random processes are generated using the FFT-method [17].

The identification performance is evaluated by averaging the mean squared deviation (MSD) over 50 simulation trials. The MSD in every simulation trial is computed as:

$$
\operatorname{MSD}(i)=\frac{\|\mathbf{h}(i)-\hat{\mathbf{h}}(i)\|_{2}^{2}}{E_{h}},
$$

where $E_{h}=(1 / N) \sum_{i=1}^{N}\|\mathbf{h}(i)\|^{2}$ and $N=10^{4}$ is a number of samples after the algorithm convergence; the MSD is averaged over these $N$ samples.

In the simulations, assuming that the sampling rate is $1000 \mathrm{~Hz}$, we use $f_{\max }=1 \mathrm{~Hz}$, which is typical for underwater acoustic channels [18]. The signal to noise ratio (SNR) is set to $25 \mathrm{~dB}$.

Fig. 1 compares the MSD performance of the FRLS and SRLS algorithms against the window length $M$. The classical SRLS algorithm achieves an MSD performance of $-15.2 \mathrm{~dB}$ when $M=71$. Its non-causal version, the delayed SRLS 


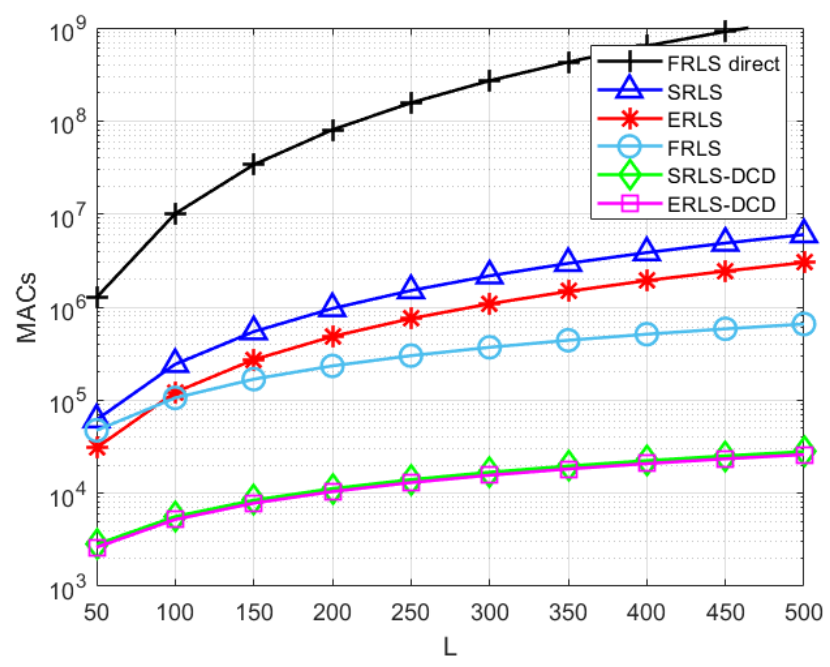

Fig. 2: Complexity comparison of the SRLS and FRLS-based algorithms; the window length is $M=3 L+1$.

TABLE II: Complexity of the adaptive algorithms

\begin{tabular}{lcc}
\hline Adaptive algorithm & MACs & FFTs \\
\hline ERLS-DCD & $\left(20+4 N_{u}\right) L+N_{u}+M_{b}$ & - \\
SRLS-DCD & $\left(24+4 N_{u}\right) L+N_{u}+M_{b}$ & - \\
FRLS & $\left(18+4 N_{u}\right) L+16 M+M_{b}$ & 7 \\
SRLS & $24 L^{2}+32 L$ & - \\
ERLS & $12 L^{2}+16 L$ & - \\
FRLS direct & $2 M L(L+1)+4 L^{3}$ & - \\
\hline
\end{tabular}

(SRLSd) algorithm, reduces the MSD to $-20.1 \mathrm{~dB}$; note that the SRLSd algorithm is equivalent to the FRLS algorithm with a symmetrical rectangular window. With the Hanning window in the FRLS (direct) algorithm, the MSD is further reduced to $-22.1 \mathrm{~dB}$, which is $2.0 \mathrm{~dB}$ improvement compared to the SRLSd algorithm and $6.9 \mathrm{~dB}$ improvement compared to the classical SRLS algorithm. Note that in this scenario the lowest MSD provided by the ERLS algorithm is $-15.8 \mathrm{~dB}$, achieved with the forgetting factor $\lambda=0.94$; this is close to the minimum MSD of the classical SRLS algorithm.

With $N_{u}=8$ updates, the DCD-based versions of these algorithms show the MSD performance close to that of their original versions, except at low values of $M$. At low $M$, the system of equations becomes ill-conditioned and the DCD solution with its implicit regularization shows a better performance. The minimum MSD of the FRLS algorithm is $-21.9 \mathrm{~dB}$ when $M=181$.

The algorithm complexities at every time instant are summarized in Table II. They are shown against $L$ in Fig. 2, where the FFT complexity is counted as $4(L+M) \log _{2}(L+M)$ MACs [19]. The ERLS and SRLS algorithms implemented using DCD iterations [4] have the lowest complexity. Note that the SRLS (SRLS-DCD) and SRLSd (SRLSd-DCD) algorithms have the same complexity. The FRLS complexity is higher than that of the ERLS-DCD and SRLS-DCD algorithms, and this is the payment for the improved performance. However, the FRLS algorithm has a comparable or lower complexity than the classical SRLS algorithm, and significantly lower complexity than the direct implementation of the solution

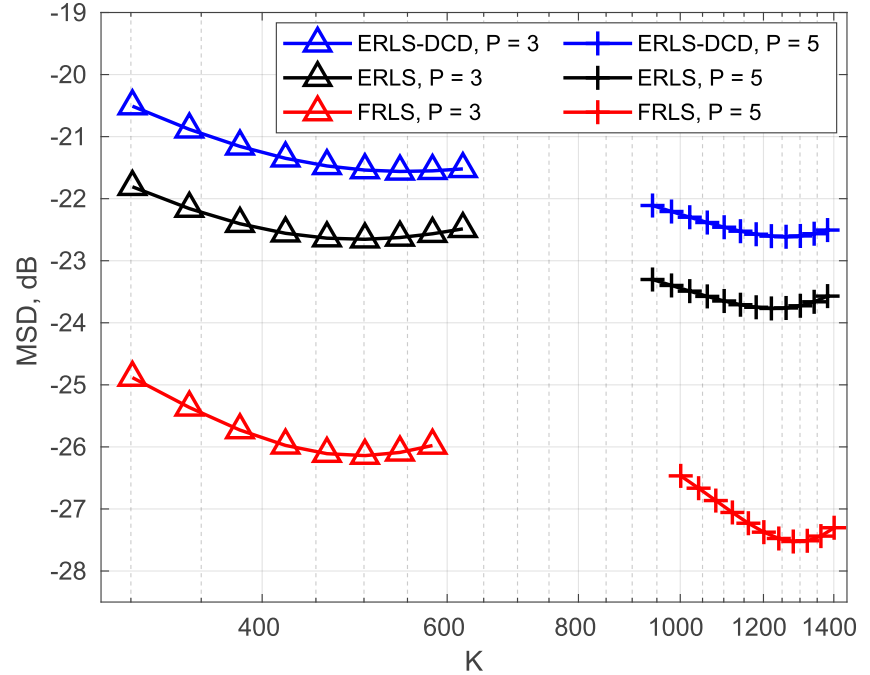

Fig. 3: The performance of the fLBF algorithms in fastvarying channel, $K$ is the approximation window length used for post-filtering.

in (2), especially for high $L$.

We now show that the FRLS algorithm with the Hanning window as a pre-estimator in the fLBF algorithm can significantly improve the fLBF identification performance compared to the use of the ERLS algorithm for this purpose. As recommended in [20], the forgetting factor of the ERLS algorithm is set to $\lambda=\max (0.9,1-2 / L)=0.96$. In the FRLS algorithm, we set $M=3 L+1=151$. We consider the cases with $P=3$ and $P=5$ basis functions. Fig. 3 shows the MSD performance of the fLBF algorithm against $K$. The original fLBF algorithm (with the ERLS pre-estimator), provides an MSD of $-22.7 \mathrm{~dB}$ and $-23.8 \mathrm{~dB}$ for $P=3$ and $P=5$, respectively. The FRLS pre-estimator with $N_{u}=8 \mathrm{DCD}$ iterations shows an improvement of, respectively, $3.5 \mathrm{~dB}$ and $3.8 \mathrm{~dB}$ against the classical-ERLS pre-estimator. Against the ERLS-DCD pre-estimator, this improvement is higher, $4.6 \mathrm{~dB}$ and $4.9 \mathrm{~dB}$, respectively.

\section{CONClusion}

We have proposed the FRLS algorithm that allows RLS adaptive filtering with any finite-length window in the cost function (1) at a complexity comparable or lower than that of the classical ERLS and SRLS algorithms. As demonstrated by the simulation results, the use of a non-uniform window in the FRLS algorithm, such as the Hanning window, significantly improves the identification performance compared to that of the classical ERLS and SRLS algorithms in time-varying scenarios.

\section{REFERENCES}

[1] S. Haykin, Adaptive Filter Theory. Prentice Hall, 2002.

[2] A. H. Sayed, Fundamentals of adaptive filtering. John Wiley \& Sons, 2003.

[3] J. Cioffi and T. Kailath, "Fast, recursive-least-squares transversal filters for adaptive filtering," IEEE Transactions on Acoustics, Speech, and Signal Processing, vol. 32, no. 2, pp. 304-337, 1984. 
[4] Y. V. Zakharov, G. P. White, and J. Liu, "Low-complexity RLS algorithms using dichotomous coordinate descent iterations," IEEE Transactions on Signal Processing, vol. 56, no. 7, pp. 3150-3161, 2008.

[5] R. B. Blackman and J. W. Tukey, "The measurement of power spectra from the point of view of communications engineering-Part I," Bell System Technical Journal, vol. 37, no. 1, pp. 185-282, 1958.

[6] F. J. Harris, "On the use of windows for harmonic analysis with the discrete Fourier transform," Proceedings of the IEEE, vol. 66, no. 1, pp 51-83, 1978.

[7] M. Niedźwiecki, M. Ciołek, and Y. Kajikawa, "On adaptive covariance and spectrum estimation of locally stationary multivariate processes," Automatica, vol. 82, pp. 1-12, 2017.

[8] Y. V. Zakharov and T. Tozer, "Multiplication-free iterative algorithm for LS problem," Electronics Letters, vol. 40, no. 9, pp. 567-569, 2004.

[9] J. Liu, Y. V. Zakharov, and B. Weaver, "Architecture and FPGA design of dichotomous coordinate descent algorithms," IEEE Transactions on Circuits and Systems I: Regular Papers, vol. 56, no. 11, pp. 2425-2438, 2009.

[10] M. Niedźwiecki and M. Ciołek, "Generalized Savitzky-Golay filters for identification of nonstationary systems," Automatica, vol. 108, p. 108477, 2019.

[11] M. J. Niedźwiecki, M. Ciołek, and A. Gańcza, "A new look at the statistical identification of nonstationary systems," Automatica, vol. 118, p. 109037, 2020.

[12] L. Shen, Y. Zakharov, B. Henson, N. Morozs, and P. D. Mitchell, "Adaptive filtering for full-duplex UWA systems with time-varying selfinterference channel," IEEE Access, vol. 8, pp. 187 590-187 604, 2020.

[13] L. Shen, Y. Zakharov, L. Shi, and B. Henson, "BEM adaptive filtering for SI cancellation in full-duplex underwater acoustic systems," Signal Processing, vol. 191, pp. 108366-108 378, 2022.

[14] M. K. Tsatsanis and G. B. Giannakis, "Modelling and equalization of rapidly fading channels," International Journal of Adaptive Control and Signal Processing, vol. 10, no. 2-3, pp. 159-176, 1996.

[15] A. M. Sayeed and B. Aazhang, "Joint multipath-Doppler diversity in mobile wireless communications," IEEE Transactions on Communications, vol. 47, no. 1, pp. 123-132, 1999.

[16] Y. Zakharov and V. Kodanev, "Multipath-Doppler diversity of OFDM signals in an underwater acoustic channel," in Proceedings of IEEE Int. Conf. Acoustics, Speech, and Signal Processing (ICASSP), vol. 5, 2000, pp. 2941-2944.

[17] D. B. Percival, "Simulating Gaussian random processes with specified spectra," Computing Science and Statistics, vol. 24, pp. 534-538, 1992.

[18] M. Stojanovic and J. Preisig, "Underwater acoustic communication channels: Propagation models and statistical characterization," IEEE communications magazine, vol. 47, no. 1, pp. 84-89, 2009.

[19] P. Duhamel and H. Hollmann, "Split-radix FFT algorithm," Electronics letters, vol. 20, no. 1, pp. 14-16, 1984.

[20] M. J. Niedźwiecki, M. Ciołek, A. Gańcza, and P. Kaczmarek, "Application of regularized Savitzky-Golay filters to identification of timevarying systems," Automatica, vol. 133, p. 109865, 2021. 